\title{
Video Article \\ Dried Blood Spot Collection of Health Biomarkers to Maximize Participation in Population Studies
}

\author{
Michael W. Ostler ${ }^{1}$, James H. Porter ${ }^{1,2}$, Orfeu M. Buxton ${ }^{1,2,3,4}$ \\ ${ }^{1}$ Center for Population and Development Studies, Harvard School of Public Health \\ ${ }^{2}$ Department of Medicine, Brigham and Women's Hospital \\ ${ }^{3}$ Division of Sleep Medicine, Harvard Medical School \\ ${ }^{4}$ Department of Biobehavioral Health, Pennsylvania State University
}

Correspondence to: Orfeu M. Buxton at Orfeu@PSU.edu

URL: https://www.jove.com/video/50973

DOI: doi:10.3791/50973

Keywords: Medicine, Issue 83, dried blood spots (DBS), Biomarkers, cardiometabolic risk, Inflammation, standard precautions, blood collection

Date Published: 1/28/2014

Citation: Ostler, M.W., Porter, J.H., Buxton, O.M. Dried Blood Spot Collection of Health Biomarkers to Maximize Participation in Population Studies. J. Vis. Exp. (83), e50973, doi:10.3791/50973 (2014).

\section{Abstract}

Biomarkers are directly-measured biological indicators of disease, health, exposures, or other biological information. In population and social sciences, biomarkers need to be easy to obtain, transport, and analyze. Dried Blood Spots meet this need, and can be collected in the field with high response rates. These elements are particularly important in longitudinal study designs including interventions where attrition is critical to avoid, and high response rates improve the interpretation of results. Dried Blood Spot sample collection is simple, quick, relatively painless, less invasive then venipuncture, and requires minimal field storage requirements (i.e. samples do not need to be immediately frozen and can be stored for a long period of time in a stable freezer environment before assay). The samples can be analyzed for a variety of different analytes, including cholesterol, C-reactive protein, glycosylated hemoglobin, numerous cytokines, and other analytes, as well as provide genetic material. DBS collection is depicted as employed in several recent studies.

\section{Video Link}

The video component of this article can be found at https://www.jove.com/video/50973/

\section{Introduction}

Biomarkers can provide valuable information about biological processes ranging from normal to pathogenic that may contribute to clinically identifiable disease ${ }^{1}$. In research settings, dried blood spots (DBS) are becoming increasingly preferred to venipuncture as a method for collecting biomarkers in whole blood. DBS collection is simple, relatively painless, less invasive then venipuncture, and requires minimal storage requirements (i.e. samples do not need to be immediately frozen and can be stored for a long period of time in a stable freezer environment before assay). DBS samples can be tested for a variety of different analytes, including cholesterol, C-reactive protein, glycosylated hemoglobin, Epstein-Barr virus antibodies, and several cytokines.

Venipuncture has long been the gold standard for the collection of biomarkers in blood. However, obtaining, storing, and shipping blood samples requires adherence to a number of protocols and health codes, which may not be possible in certain research protocols. Phlebotomists must be officially certified for venipuncture, while DBS sampling procedures are simpler and do not require official certification. Venous blood collection also requires more equipment than does DBS collection (i.e. needles, tubes, tourniquets), and more post-collection effort. Venipuncture samples must be processed (i.e. serum or plasma extracted from the blood sample via centrifugation or standing at room temperature for a given period of time) and frozen between $-20^{\circ} \mathrm{C}$ and $-70{ }^{\circ} \mathrm{C}$ immediately in order to prevent the degradation of analytes, while DBS samples must simply be allowed to air dry and then may be stored at room temperature for a week or more depending on the analyte, before transfer to a freezer ${ }^{2}$. DBS samples are less expensive to collect than venipuncture samples for reasons described above. DBS samples also take up less space, reducing shipping and storage costs prior to assay.

The use of saliva as biomarker has become key in biobehavioral studies. Saliva offers a simple, noninvasive method to collect biomarkers and has become the standard for field work aimed at measuring cortisol or cotinine ${ }^{3-5}$. Urine is another specimen that can be collected noninvasively and can be used in population level studies for assays of melatonin or for toxicology. While both urine and saliva offer straight forward collection procedures that are able to be completed by participants themselves, not all analytes can be measured in these media.

DBS offer a simple, cost effective, alternative to venipuncture and are becoming an increasingly implemented research tool in field and population studies ${ }^{6}$. 


\section{Protocol}

The following protocol has been approved by the applicable institutional review board and was conducted in accordance with the Declaration of Helsinki.

\section{Before Beginning: Standard Precautions}

Ensure all sample collection staff have passed the NIOSH required Blood Borne Pathogen (BBP) Training.

\section{Engineering Controls}

1. Use Food and Drug Administration approved sharps disposal containers to dispose of used sterile, single-use lancets. Use a properly labeled biowaste barrel for any other material that comes in contact with possibly infected material.

2. Do not overfill the sharps container. Only fill a sharps container to $3 / 4$ capacity before replacing the container to prevent injury. There is an indicator for compartmental fullness on every sharps container.

3. Only dispose of sharps in the sharps container. Do not use the sharps container for any other waste.

4. Dispose of any nonsharp material that comes in contact with potential BBP in a properly labeled biowaste barrel.

2. Personal Protective Equipment (PPE)

1. Always properly use PPE to protect against infection from BBP. PPE used during collection of DBS include gloves, lab coats, eye protection, long pants and closed-toed shoes.

2. Wear gloves to handle blood or anything that has contacted blood or other potentially infectious materials (OPIMs). Gloves should not be too tight nor too loose, as this may impede the process of "milking" (to be described later). Do not wear rings while collecting blood spots as they could puncture the glove.

3. Wear a lab coat to protect skin and clothing from blood and OPIMs. A lab coat that has come in contact with a BBP should be laundered in a $10 \%$ bleach/water solution prior to reuse. Regardless of exposure, the lab coat should be laundered regularly to keep it clean.

4. Wear closed-toed shoes to protect feet against possible contact with blood and OPIMs.

5. If desired, wear goggles to protect against possible contact with blood and OPIMs due to splatter.

6. To maintain the cleanliness of a workspace, remove all PPE before leaving the work area to avoid contaminating items outside of the lab area.

3. Work Practice Controls

1. Wash hands with soap and warm water for $20 \mathrm{sec}$ before and after physical contact with each participant. Use a towel to turn off the running water. If a sink is not available, use a hand sanitizer of at least $60 \%$ alcohol.

2. Regardless of the opportunity to wash hands, use alcohol hand sanitizer before applying and after removing gloves. Use enough sanitizer to cover all surfaces of both hands and rub hands together until dry. Do not rinse or wipe hands dry with a towel as this will reduce the efficacy of the sanitizer.

3. Do not eat or drink or apply makeup or contact lenses in blood collection areas. Personal exposure to BBPs could occur through these paths that are direct routes to mucous membranes.

4. Do not handle biohazardous material without gloves and do not handle nonhazardous material with gloves that have previously touched biohazardous material.

4. Proper Housekeeping

Good housekeeping will continue to help protect against exposure to a BBP and will prevent cross-contaminating the environment.

1. Use virucidal microbial disinfectant wipes to properly clean areas contaminated during DBS collection.

2. Keep biohazardous or contaminated waste separate from ordinary trash; contaminated materials are those that have visible blood or OPIM on them.

\section{Exposure Response}

If exposed to a BBP, respond in the following way.

1. Wash the exposed area immediately and thoroughly with soap and water. Quick action can reduce the risk of infection.

2. Identify the source and route of the exposure to be able to explain the situation to the field supervisor and/or the responding physician.

3. Notify the on-call supervisor immediately after the exposure occurs. It is emphasized to get support from a field supervisor to understand the level of risk for infection so it can be assessed if immediate medical attention is necessary. Guidelines laid out in specific Exposure Control Plans (ECPs) must be provided to all those that are in danger of being exposed to BBP.

\section{Setup of Collection Room and Materials}

1. Select a sample collection location that is NOT intended for eating, such as a breakroom, and that is free of all unrelated project equipment. One does not want to be in an area that is crowded; it is important to have ease of movement. The following describes how to set up the dried blood-spot collection (lab) area.

2. Set up the biohazard waste barrel and line the interior with a biohazard waste bag. The waste barrel should be accessible while seated.

3. Clean the collection area thoroughly with disinfecting wipes or similar product. Dispose of all material used to clean the lab area in the biohazard waste barrel. Wear gloves during this step to protect skin from exposure to harmful chemicals.

4. Replace gloves and set up remaining collection supplies.

1. Place gloves and alcohol hand sanitizer on the collection table. 
2. Place the sharps container within reach of the collector's chair. The sharps container should be handled with gloves at all times and then properly disposed of when full.

3. Place all remaining materials required for DBS collection on a protective underpad in the collection area. Include a filter paper collection card, a few lancets, alcohol pads, gauze pads, and Band-Aids, but not too many so the area is cluttered. Do not set up more than what might be used for each participant.

\section{DBS Sample Cellection}

Always wear PPE (lab coat, gloves, long pants, closed-toed shoes and if desired, safety glasses) during collection and place a protective underpad (chux) on lap to protect one's street clothes.

1. Preparing the participant:

1. After obtaining consent, ask the participant if he/she would prefer to use the left or right hand for collection and select a finger for collection. Avoid heavily calloused or damaged (lacerated) fingers and any ringed fingers. Typically the pinky and thumb are not selected so as to minimize participant pain and facilitate collection, respectively. The sides of the finger tips are used as the collection site for consistency and ease of collection.

2. Have the participant rub their hands together to warm their hands and stimulate blood flow. A hand warmer may be used, or the participant may run their hands under warm water if a sink is available. Another technique to stimulate blood flow would be to have the participant alternately clench and relax the target hand, then rest fully prior to the fingerstick.

3. Clean the selected finger with an alcohol pad and allow it to fully dry. Repeat with a second alcohol pad to fully remove any contaminates or hand lotions. The finger must be completely dry before proceeding with the finger stick or the alcohol will contaminate the blood sample. As with all used collection materials, immediately dispose of the alcohol wipes in the biohazard waste container.

2. Preparing the puncture site on the participant's finger:

1. Secure the participant's arm to prevent movement during collection and to relax the arm and hand muscles. Rest the participant's elbow on the edge of the table or on their lap. Keep the participant's hand positioned comfortably below heart level and their muscles relaxed to facilitate blood flow.

2. Grasp the chosen finger between thumb and index finger and gently milk the finger by slowly and firmly squeezing the finger while moving the grasp from palm to fingertip to pull blood towards the fingertip.

3. Press the micro-lancet firmly against the participant's finger perpendicular to the fingerprint and discharge the lancet. Do not puncture the center of the finger or close to the nail bed; instead aim between those two points, on the ulnar side of the finger. Immediately dispose of the used lancet in the sharps container.

3. Collecting the blood spots:

1. Wipe the first drop of blood away with a sterile gauze pad to remove the tissue fluid from the sample and immediately dispose of the gauze into the biohazard waste barrel.

2. While maintaining the participant's hand below their heart with a downward angle, gently milk the hand starting at the wrist and work down to the base of the finger to produce blood. Do not just squeeze the finger, as this will reduce blood flow. Milk the finger until a large drop is hanging from the fingertip.

3. Collect spots using the collection paper.

1. Carefully position the collection paper below the finger and allow the drop to fall of its own weight. This allows collection of blood spots of maximum volume. If blood pools on the filter paper, i.e. "beads", gently tap the card to encourage blood to saturate into the paper.

2. If the blood is too viscous or drops are too small to fall of their own weight, slowly bring the collection paper into contact with the blood drop to absorb the blood away from the finger via capillary action without touching the finger. If the finger touches the collection paper, move to another spot on the paper as that collection area has been compromised. The participant's finger should never touch the filter paper.

3. Do not place blood drops on top of one another, even If a blood spot does not fill the collection circle.

4. Do not let the blood run down the finger or onto the fingernail as this makes collection of large spots more difficult.

4. If the participant's blood clots any time during the collection process, ask permission to prick another finger; then clean and bandage the initial puncture site and start over if the request is accepted.

1. To prevent clotting, continue milking the participant's finger to keep the blood flowing or try firmly wiping the puncture with sterile gauze to disrupt clot formation.

Note: Subsequent finger-sticks may be necessary for a few reasons: A cold room may cause more rapid clotting; also the viscosity of participants' blood may not be ideal, or poor milking techniques can also impact blood flow. Hand warmers, or warm running water, used prior to puncture can reverse the effect of a cold room.

5. Once the DBS have been collected, or if the participant does not want subsequent finger-sticks, clean the puncture site of any extraneous blood.

6. Bandage the puncture site to prevent potential contamination once the participant leaves the sample collection area.

4. FI Quality Control (QC) assessment:

Upon completing sample collection, assess the samples for quality. Ensure spots are 1) at least 0.375 in in diameter as measured from the back of the collection area; 2) adequately soaked through the collection paper so as to be of similar size on both sides of the filter paper, spots not meeting this criterion indicate collection via contact of the finger to the collection paper and likely smearing; 3 ) not in contact with one another (overlapping areas of spots are not assayable).

5. Completing the sample collection 
1. Following guidelines described in step 1.1, dispose of any remaining contaminated collection materials, including any items that may have been in contact with potentially infectious materials.

2. Stand the collection paper on a protective underpad to dry. Space the collection cards to allow good airflow between them. The minimum amount of time to air dry is $15 \mathrm{~min}$, preferably at least $30 \mathrm{~min}$. To allow maximum air flow, it is important to fold the back of the collection card in a way that it can stand freely and provide air flow behind the samples on the collection paper.

3. With all steps complete, remove PPE.

6. Response to a subject feeling faint or not well

Although unlikely, a participant may become dizzy, lightheaded, or faint during or after the finger stick. Be alert for signs such as pallor, perspiration on the face and forehead, complaints of blurring vision, drooping or fluttering eyelids, or complaints of nausea. The onset of these symptoms is often sudden; sample collectors must be prepared to respond quickly if any of the symptoms are visible. If any of these symptoms are present at any point during the procedure, react according to the following procedures.

1. If a participant is feeling faint, lightheaded, dizzy, or shows any signs of fainting, take the following actions:

1. Stop the procedure.

2. Take care that the participant does not fall or become injured.

3. Calmly reassure the participant and if necessary, ask the participant to bend at the waist and put his/her head between his/her legs. If mobility is limited and the participant can be moved, an adjacent cushioned location can be used to rest.

4. Have the participant rest for $10 \mathrm{~min}$.

5. Resume the procedure if the participant consents to continue.

2. If a participant faints, take the following actions:

1. Stop the procedure.

2. Take care that the participant does not fall or become injured.

3. Have the participant lie on his/her back as quickly as possible with feet elevated. The participant will be instructed to lie down directly from the seated position without standing up.

4. Ask the participant to loosen any tight clothing

5. Have the participant rest for 10 min.

6. Blood spot collection should not continue.

7. If the participant does not respond after $1 \mathrm{~min}$, call 911

\section{Breaking down the lab area:}

Once the collection of blood spots has been completed, the collection area must be broken down and the area decontaminated to make certain that passer-bys will not be infected by BBP. The blood collection area, the equipment containers, collection materials, and waste containers must be clean of BBP. As always, wear PPE when handling potentially infectious materials.

1. Remove from the collection area and repack all uncontaminated materials.

2. Dispose of any remaining contaminated waste material into the biowaste barrel.

3. Close the sharps container and store it in an area that provides protection from contamination. If the sharps container is $3 / 4$ full, appropriately dispose of it and replace with a new sharps container.

4. Wipe the entire sample collection area with virucidal microbial disinfecting wipes.

5. Once all biohazardous material has been disposed of, the biohazard bag can be autoclaved. When removing the bag from the waste barrel, be cautious to only touch the outside of the bag and to seal the bag tightly. It is also good practice to use virucidal microbial wipes to clean the exterior of all equipment to prevent cross-contamination.

\section{Training to Collect DBS}

Verify any dried blood spot collector is certified by a qualified individual before performing the procedure on patients or research subjects. Ensure collectors demonstrate respect for participants, confidence and proficiency in the use of PPE and of blood collection supplies, in the collection of spots of sufficient volume, and in completing the procedure in a timely and efficient manner, ideally in under $10 \mathrm{~min}$.

\section{Sample Packaging and Storage}

1. Ensure DBS samples have dried for a minimum 15 min before they are bagged for storage, a saturated card may take 30 min or longer to adequately dry. Close the collection card cover, ensuring the sample is completely dry to prevent smearing and contaminating the sample.

2. Take a biohazard specimen bag and place a desiccant pouch into the specimen bag. Place the DBS sample into the biohazard specimen bag. Always wear gloves when handling DBS samples. Do not allow the blood samples to touch the outside of the specimen bag.

3. Place each sample in its own biohazard sample bag with a desiccant packet. Flatten the bag to remove excess air and then seal the bag tightly. Place the bagged sample in a storage cooler for transport or temporary storage. Avoid elevated temperatures.

4. Freeze or assay DBS samples within two weeks of collection, as DBS samples may be typically stored at room temperature for two weeks without significant degradation depending on analyte ${ }^{7,2}$.

\section{Sample Transportation and Shipment}

1. Unless known to contain infectious agents, ship dried blood spot samples as exempt human specimens under U.S. Department of Transportation regulations and in accordance with Part 2, Chapter 6, §6.3.2.3.6 of the International Civil Aviation Organization (ICAO) Technical Instructions ${ }^{8}$. 
2. If frozen, ship samples overnight on dry ice with the requisite extra precautions for hazardous substances, or with gelpaks frozen to $-80{ }^{\circ} \mathrm{C}$. Ship on Monday-Wednesday to avoid thaw in transit due to delays or weekends/holidays, with confirmation from the receiving site that they are expecting and ready to receive and freeze samples.

\section{Quality Control Assessment}

1. Following collection and prior to assay (preferably prior to freezing in order to minimize the number of freeze-thaw cycles) assess samples for quality.

2. Include the following criteria in assessment of sample quality; proper packaging, color-coded desiccant remaining intact (dry), saturation of spots, size of spots, proper collection methods including spots not overlapping or smeared on card indicating contact with subject finger.

\section{Freezer Storage}

Although degradation times differ by analyte, freeze DBS samples within two weeks of collection for best results. Freeze samples with desiccant in a laboratory-grade freezer. As with any biological specimen, minimize freeze-thaw cycles, although some assays in DBS samples have shown no evidence of degradation in up to six freeze-thaw cycles $^{9-11}$.

\section{Representative Results}

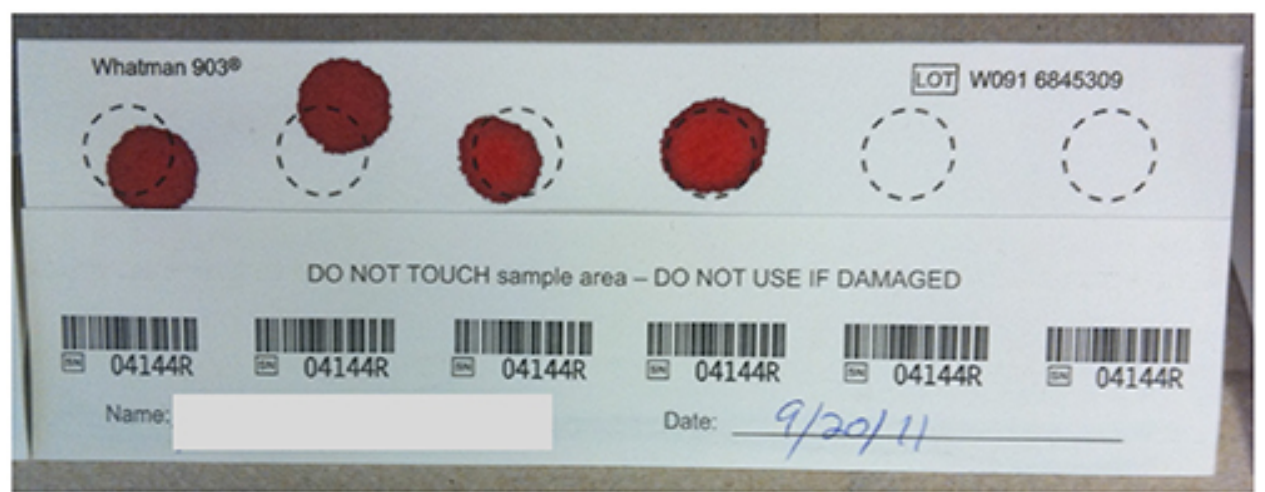

Figure 1. Dried blood spot sample. A collection card is shown with four, valid collected spots in the process of drying. Click here to view larger image. 


\section{BAD}

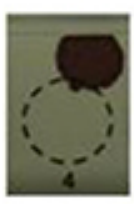

\section{Blood is out of}

designated area

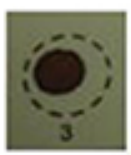

Too small

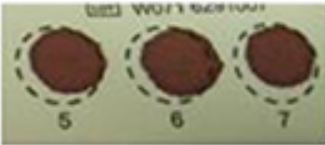

\section{Overlapping}

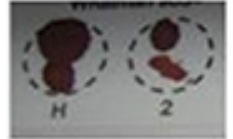

Too small and

overlapping

Figure 2. Good and bad dried blood spot samples. Results of successful (right) and unsuccessful (left) DBS collection are shown. Click here to view larger image. 

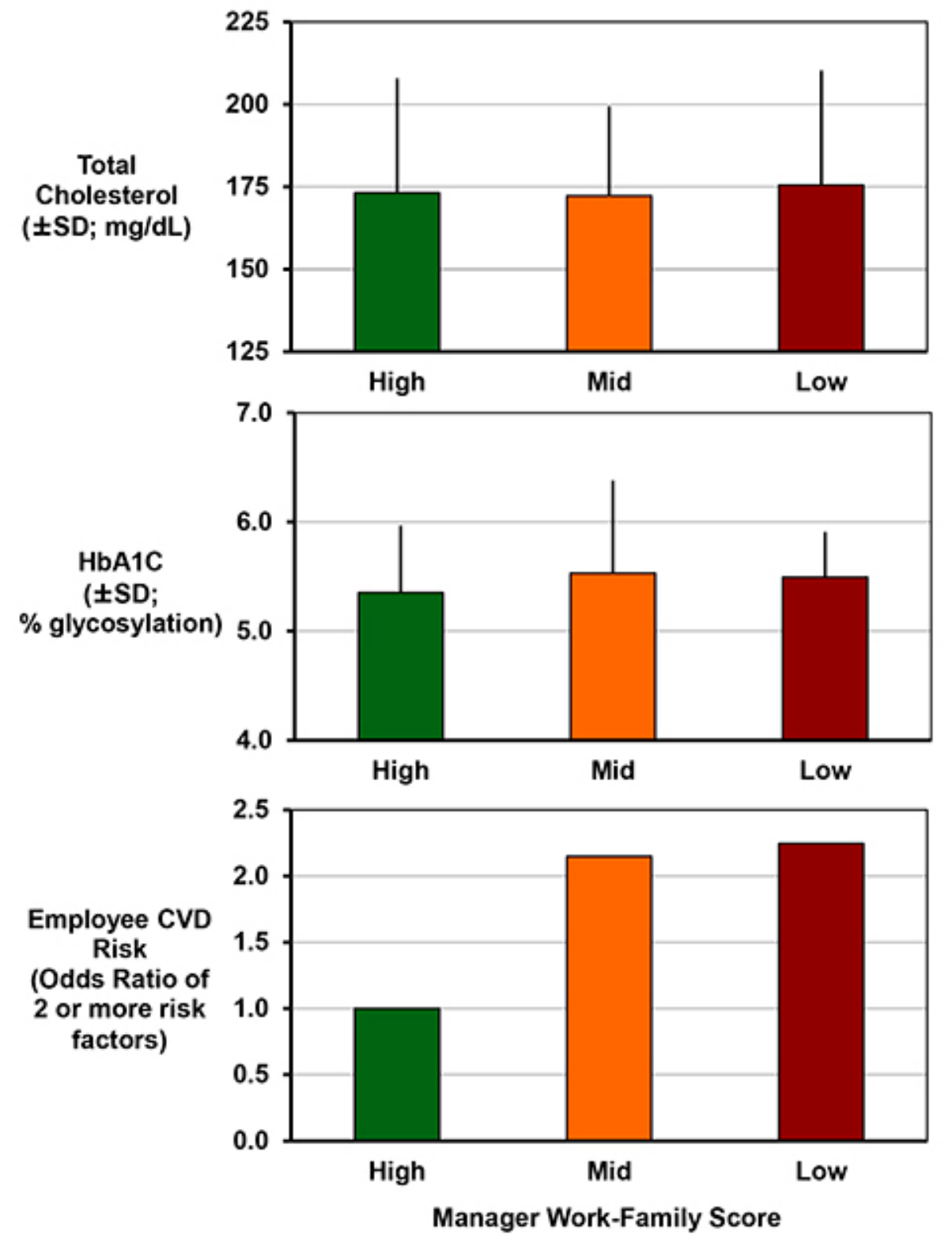

Figure 3. Managers' attitudes $\&$ practices associated with employee cardiometabolic disease risk $^{6}$. This figure has been modified from Berkman, L. F., Buxton, O. M., Ertel, K. \& Okechukwu, C. Manager's practices related to work-family balance predict employee cardiovascular risk and sleep duration in extended care settings. Journal of Occupational Health Psychology 115, 316-329 (2010). Employees exposed to managers with mid or low work-family scores of openness and creativity for helping with their employees' work and family need, have statistically significant increased odds of having two or more cardiovascular risks (current smoker, obesity, high blood pressure or hypertension, high cholesterol, and high glycosylated hemoglobin ( $\mathrm{Hb} \mathrm{A1C}$ ) or diabetes diagnosis). Top and middle panels depict mean values \pm standard deviations for total cholesterol assessed by dried blood spot and $\mathrm{Hb} \mathrm{A1C}$ assessed by point of care device (which can also be reliably measured by dried blood spots). Bottom panel shows cardiovascular disease risk by manager score. Analysis restricted to nurses and other employees providing direct care indicate that exposure to managers with low creativity and openness related to work-family issues may be particularly important for these individuals (OR for low W-F score $=6.4, p=0.01$ ). All models control for age, gender, wage, education, race/ethnicity, and worksite. Click here to view larger image.

\section{Discussion}

Biomarkers are extremely useful tools across multiple disciplines, including medicine, cell biology, genetics, psychology, sociology, demography, and social epidemiology. Within each discipline, early phase validity and reliability biomarker research was used to uncover both hidden disease risk and provide longitudinal prediction of health outcomes. In these instances, biomarkers that are accurate, durable (i.e. they can survive the lag between sample collection and implementation of the lab assay to extract the biomarker), and versatile (i.e. useful in a variety of models) are required. 
While DBS collection is a relatively straightforward procedure, several techniques can help maximize volume of collected spots. Before collection begins, ensuring a participant's hand is warm, and therefore that blood vessels are dilated, will help blood flow once the finger is punctured. Using hand warmers or having a participant run their hands under warm water can help with this process. Milking the finger before collection will pull blood to the finger tip and warm the hand to help blood flow. Milking is a process that improves with practice. Collectors should use whatever technique they find most comfortable and effective, but the most common approach involves milking the participant's hand with the collector's opposite hand. This method will allow the collector's thumb to work deep into the participant's palm while the opposite hand can stabilize the participant's wrist. As an example, if one were collecting blood from a finger on the participant's left hand, the collector would stabilize the participant's wrist with their left hand and milk with their right hand. Common mistakes made while milking include not using a firm grip, pulling the skin above the hand rather working into the muscles to pull blood towards the finger tip. It is also important to avoid pinching the finger with the milking hand or tightly gripping the participant's wrist with the nonmilking hand, both of which impede blood flow.

When puncturing the finger, make the puncture as deep as possible by firmly pressing the lancet into the finger before discharge. A shallow puncture results in less blood flow and quicker clotting. After puncturing the finger, working quickly is key. Thin blood leads to easier collection and larger spots. After puncture, the blood becomes more and more viscous until clotting. The less time required to collect the blood spots on the card the greater their volume.

Ideally the participant's blood will be thin enough that drops will fall of their own weight, but this will not be the case for all participants, as some people's blood is thicker or clots faster than others. If the blood is too thick to fall from the finger, the filter card must be brought to the blood for collection. When bringing the card to the blood it is important to hold the card in contact with the drop while it is hanging from the finger for as long possible, rather than just dabbing the blood to the card. This will help pull blood from the finger to maximize the size of the spot. This technique requires steady hands to maintain contact between the blood and the card. Using both hands, one to steady the participants hand and one to hold the card, and keeping both hands in contact with one another helps to stabilize the card and the blood together.

Advanced research areas, such as cardiometabolic risk assessment, have long relied upon biomarkers such as C-reactive protein (CRP), insulin, and glycosylated hemoglobin ( $\mathrm{HbA1C}$ ) for early risk detection and prevention efforts, as well as biomarkers of "hard endpoints" for cardiovascular disease (CVD) risk and CVD-associated mortality ${ }^{12}$. Clinical epidemiology frequently assesses biomarkers to prospectively predict cardiometabolic risk outcomes such as heart disease and Type II diabetes. Perhaps the most widely used of all biomarkers is the Framingham risk score, a composite that includes blood pressure, HDL cholesterol levels and total cholesterols levels that has demonstrated utility in predicting CVD ${ }^{13}$. However, the Framingham score has experienced some technical controversies, and has limitations in studies focused on linking social and environmental factors to health, suggesting the need for more accurate and specific biomarkers related to cardiovascular research.

There are a number of commercially available kits using ELISA or similar techniques available for the assay of DBS samples. Necessary for the assay of DBS samples are the use of specific and optimized elution steps to bring the dried samples into solution ${ }^{2}$. This is an expanding area of research as assays for more analytes are currently being developed ${ }^{1}$. Among the assays already validated and being widely used are cholesterol $^{2}, \mathrm{CRP}^{10,14,15}, \operatorname{IgE} 16, \mathrm{EBV}^{9,15}$, leptin ${ }^{17}$, and cyotkines ${ }^{18}$. There are a number of laboratories specializing in the assay of DBS, including the University of Washington Department of Laboratory Medicine (UW Lab Med) Biomarker Laboratory (Director: Mark Wener).

Bearing in mind that a panel of biomarkers, rather than a lone biomarker, is most useful in the fields of cardiovascular and diabetes research, biomarkers need to be considered along with a variety of contextual factors in order to maximize their utility. Biomarker research can be expensive however, both financially and in time for data collection. Thus, while it is vital to choose biomarkers that are accurate, durable, and versatile, work and family biomarker research must be rooted in practicality by selecting biomarkers that are inexpensive, appropriate, and can be collected quickly with ease. Furthermore, practical considerations often result in an inability to simultaneously maximize price, quality, and speed within one procedure. Herein lie the next steps in health biomarker research within field and population studies. The field of biomarker research is currently in a period of dramatic growth; however, most of the work is yet to come.

\section{Disclosures}

Orfeu Buxton has received an investigator-initiated grant from Sepracor Inc (now Sunovion; ESRC-0977, ClinicalTrials.gov Identifier NCT00900159); serves as a consultant and expert witness for Dinsmore LLC, on the Scientific Advisory Board of Matsutani America, and received consulting fees from the Wake Forest University Medical Center.

\section{Acknowledgments}

This work was supported by the Work, Family and Health Network (www.WorkFamilyHealthNetwork.org), which is funded by a cooperative agreement through the National Institutes of Health and the Centers for Disease Control and Prevention: Eunice Kennedy Shriver National Institute of Child Health and Human Development (Grant \# U01HD051217, U01HD051218, U01HD051256, U01HD051276), National Institute on Aging (Grant \# U01AG027669), Office of Behavioral and Science Sciences Research, the National Heart, Lung and Blood Institute (R01HL107240), and National Institute for Occupational Safety and Health (Grant \# U01OH008788, U01HD059773). Grants from the William T. Grant Foundation, Alfred P Sloan Foundation, and the Administration for Children and Families have provided additional funding. The contents of this publication are solely the responsibility of the authors and do not necessarily represent the official views of these institutes and offices.

\section{References}

1. Buxton, O. M., Klein, L. C., Whinnery, J., Williams, S. \& McDade, T. in New Frontiers in Work and Family Research. eds J.G. Grzywacz \& E. Demerouti. Psychology Press, Taylor \& Francis Group, Routledge (In Press).

2. McDade, T. W., Williams, S. \& Snodgrass, J. J. What a drop can do: dried blood spots as a minimally invasivemethod for integrating biomarkers into population-based research. Demography. 44, 899-925 (2007). 
3. Kirschbaum, C., Read, G. F. \& Hellhammer, D. H. Assessment of hormone and drugs in saliva in biobehavioral research. Hogrefe \& Huber Publishers (1992).

4. Piazza, J. R., Almeida, D. M., Dmitrieva, N. O. \& Klein, L. C. Frontiers in the use of biomarkers of health in research on stress and aging. J. Gerontol. Series B Psychol. Sci. Soc. Sci. 65, 513-525, doi:10.1093/geronb/gbq049 (2010).

5. Smyth, J. M. et al. Individual differences in the diurnal cycle of cortisol. Psychoneuroendocrinology. 22, 89-105 (1997).

6. Berkman, L. F., Buxton, O. M., Ertel, K. \& Okechukwu, C. Manager's practices related to work-family balance predict employee cardiovascular risk and sleep duration in extended care settings. J. Occup. Health Psychol. 115, 316-329 (2010).

7. Buxton, O. M., Malarick, K., Wang, W. \& Seeman, T. Changes in dried blood spot Hb A1c with varied postcollection conditions. Clin. Chem. 55, 1034-1036 (2009)

8. International Civil Aviation Organization. Technical Instructions for the Safe Transport of Dangerous Goods by Air (Doc 2984). 2009-2010 edn, Council of ICAO (2010).

9. McDade, T. W. et al. Epstein-Barr virus antibodies in whole blood spots: a minimally invasive method for assessing an aspect of cell-mediated immunity. Psychosom. Med. 62, 560-567 (2000).

10. McDade, T. W., Burhop, J. \& Dohnal, J. High-sensitivity enzyme immunoassay for C-reactive protein in dried blood spots. Clin. Chem. 50, 652-654 (2004).

11. McDade, T. W. \& Shell-Duncan, B. Whole blood collected on filter paper provides a minimally invasive method for assessing human transferrin receptor level. J. Nutr. 132, 3760-3763 (2002).

12. Gerszten, R. E. \& Wang, T. J. The search for new cardiovascular biomarkers. Nature. 451, 949-952, doi:10.1038/nature06802 (2008).

13. D'Agostino, R. B., Sr., Grundy, S., Sullivan, L. M. \& Wilson, P. Validation of the Framingham coronary heart disease prediction scores: results of a multiple ethnic groups investigation. J. Am. Med. Assoc. 286, 180-187 (2001).

14. McDade, T. W., Rutehrford, J., Adair, L. \& Kuzawa, C. W. Early origins of inflammation: microbial exposures in infancy predict lower levels of C-reactive protein in adulthood. Proc. R Soc. B. doi:10.1098/rspb.2009.1795 (2009).

15. McDade, T. W. in Measuring Stress in Humans. A Practical Guide for the Field. eds G. H. Ice \& G. D. James. Cambridge University Press, 181-207 (2007).

16. Tanner, S. \& McDade, T. W. Enzyme immunoassay for total immunoglobulin E in dried blood spots. Am. J. Hum. Biol. 19, 440-442 (2007).

17. Miller, A. A., Sharrock, K. C. \& McDade, T. W. Measurement of leptin in dried blood spot samples. Am. J. Hum. Biol. 18, 857-860 (2006).

18. Miller, E. M. \& McDade, T. W. A highly sensitive immunoassay for interleukin-6 in dried blood spots. Am. J. Hum. Biol. doi:10.1002/ajhb.22324 (2012). 